\title{
An Efficient Series Solution for Fractional Differential Equations
}

\author{
Mohammed Al-Refai, Mohamed Ali Hajji, and Muhammad I. Syam \\ Department of Mathematical Sciences, United Arab Emirates University, P.O. Box 15551, Al Ain, UAE \\ Correspondence should be addressed to Mohammed Al-Refai; m_alrefai@uaeu.ac.ae
}

Received 25 January 2014; Revised 18 February 2014; Accepted 24 March 2014; Published 6 April 2014

Academic Editor: Dumitru Baleanu

Copyright (C) 2014 Mohammed Al-Refai et al. This is an open access article distributed under the Creative Commons Attribution License, which permits unrestricted use, distribution, and reproduction in any medium, provided the original work is properly cited.

\begin{abstract}
We introduce a simple and efficient series solution for a class of nonlinear fractional differential equations of Caputo's type. The new approach is a modified form of the well-known Taylor series expansion where we overcome the difficulty of computing iterated fractional derivatives, which do not compute in general. The terms of the series are determined sequentially with explicit formula, where only integer derivatives have to be computed. The efficiency of the new algorithm is illustrated through several examples. Comparison with other series methods such as the Adomian decomposition method and the homotopy perturbation method is made to indicate the efficiency of the new approach. The algorithm can be implemented for a wide class of fractional differential equations with different types of fractional derivatives.
\end{abstract}

\section{Introduction}

During the last three decades, fractional calculus caught the attention of many researchers in differential fields of science and engineering. This is, mainly, due to the importance of noninteger order derivatives in modeling certain physical phenomena [1-4]. It turns out that, in some cases, modeling using fractional calculus is more realistic than integer calculus. This is because of the fact that the behavior of many physical phenomena depends not only upon the instantaneous state but also on the previous time history. Fractional derivative, comprising in its definition previous time history about the function, makes it more suitable for modeling systems whose evolution depends upon their current and previous states.

Recently, many researchers got interested in looking at fractional differential equations (FDEs) as new model equations for many physical problems. However, many of these such FDEs do not possess exact analytic solutions. This difficulty prompted many researchers to develop numerical schemes to find approximate solutions. Many numerical methods used to solve integer order differential equations have been adapted to treat FDEs such as the variational iteration (VIM) [5-8], the homotopy analysis method (HAM) [9-14], and the Adomian decomposition method (ADM)
[15-20], just to name a few. For a survey of recent development of methods in fractional calculus, the reader is referred to [21]. All these methods can be classified as iterative methods which produce a solution in the form of a series expansion whose terms are generated iteratively. However, for many cases, the iterative process of these methods is not easily implemented. For example, the ADM requires integration at each step to find the next iterate and the ADM requires solving a differential equation. Another approach is the upper-lower iterative method [22]. Quadrature techniques have been implemented to construct different formulations of fractional backward difference methods [23-25]. Also, fractional linear multistep methods presented for special types of the Volterra integral equation $[26,27]$ have been implemented for several types of fractional differential equations. As a result, a class of higher order backward difference methods have been obtained [28]. For more details one can refer to [29] and the references therein. Convenient and easy presentations to discretize fractional derivative of arbitrary order have been obtained in a form of triangular strip matrices; see $[30,31]$. The suggested approach leads to a significant simplification of the solutions of differential equations of fractional order.

In our present work we present a series solution method in the spirit of the Taylor series expansion for a class of 
nonlinear differential equation of fractional order. The coefficients of the series expansion are also iteratively computed but the iteration process involves only differentiation. Naturally, if the problem is of fractional order, the differentiation is also of fractional order. However, to overcome the use of fractional differentiation, we employ a transformation that allows us to use ordinary differentiation rather than fractional differentiation to recursively compute the coefficient of the series expansion. We see this as an advantage to the abovementioned methods.

In this paper, we consider the initial value problem of fractional order:

$$
\begin{gathered}
D_{t}^{\alpha} u(x, t)=u_{x x}(x, t)+h(x, t, u), \quad t>0, x \in \mathbb{R} \\
u(x, 0)=u_{0}(x), \quad x \in \mathbb{R}
\end{gathered}
$$

where $0<\alpha<1, h \in C^{\infty}\left(\mathbb{R} \times \mathbb{R}^{+} \times \mathbb{R}, \mathbb{R}\right), u_{0}(x) \in C^{\infty}(\mathbb{R}, \mathbb{R})$, and $D_{t}^{\alpha}$ is the Caputo partial fractional derivative of order $\alpha$. For $\alpha \in \mathbb{R}, n-1<\alpha \leq n, n \in \mathbb{N}^{+}$, the left Caputo fractional derivative is defined by [3]

$$
D_{t}^{\alpha} u(x, t)=\frac{1}{\Gamma(n-\alpha)} \int_{0}^{t} \frac{\partial u(x, s)}{\partial s} \frac{1}{(t-s)^{\alpha+1-n}} d s
$$

and satisfies the following properties:

(1) $D_{t}^{\alpha} f(x)=0$;

(2) $D_{t}^{\alpha} t^{r}=\frac{\Gamma(r+1)}{\Gamma(r-\alpha+1)} t^{r-\alpha}, r>n-1, r \in \mathbb{R}$;

(3) $D_{t}^{\alpha}\left(\sum_{i=0}^{m} c_{i} f_{i}(x, t)\right)=\sum_{i=0}^{m} c_{i} D_{t}^{\alpha} f_{i}(x, t)$, where $c_{0}, c_{1}$ $, \ldots, c_{m}$ are constants.

The Caputo partial fractional derivative in (3) is related to the Riemann-Liouville partial fractional integral, $I_{t}^{\alpha}$, of order $\alpha$, by

$$
D_{t}^{\alpha} f(x, t)=I_{t}^{n-\alpha} \frac{\partial^{n} f(x, t)}{\partial t^{n}}
$$

where, for $n-1 \leq \alpha<n$,

$$
I_{t}^{\alpha} f(x, t)=\frac{1}{\Gamma(\alpha)} \int_{0}^{t}(t-s)^{\alpha-1} f(x, s) d s .
$$

$I_{t}^{\alpha}$ can be considered as the inverse operator of $D_{t}^{\alpha}$ in the sense

$$
\begin{gathered}
I_{t}^{\alpha} D_{t}^{\alpha} f(x, t)=f(x, t)-\sum_{k=0}^{n-1} \frac{\partial^{k} f\left(x, 0^{+}\right)}{\partial t^{k}} \frac{t^{k}}{k !}, \\
D_{t}^{\alpha} I_{t}^{\alpha} f(x, t)=f(x, t) .
\end{gathered}
$$

In this paper, we consider $\alpha=p / q$ rational with $\operatorname{gcd}(p, q)=$ 1. The paper is organized as follows. In Section 2, we present the series solution method to problem (1) and (2). In Section 3, we present numerical results to illustrate the efficiency of the presented technique. Comparison with other methods such as the Adomian decomposition method (ADM) and the homotopy perturbation method (HPM) will be also presented in Section 3. Finally, we conclude with some remarks in Section 4.

\section{Series Method}

In this section, we present the series solution method to solve problem (1) and (2) and we give the final result for the ODE version of (1) and (2). Given the order $\alpha=p / q$, we assume that the solution $u(x, t)$ takes the form

$$
u(x, t)=\sum_{k=0}^{\infty} a_{k}(x) t^{k / q}
$$

where $u(x, 0)=u_{0}(x)$ and $a_{k}(x)$ are functional coefficients to be determined. Clearly, $a_{0}(x)=u_{0}(x)$. Formal substitution of (7) into (1) gives

$$
\begin{aligned}
D_{t}^{\alpha}\left(\sum_{k=0}^{\infty} a_{k}(x) t^{k / q}\right)= & \sum_{k=0}^{\infty} a_{k}^{\prime \prime}(x) t^{k / q} \\
& +h\left(x, t, \sum_{k=0}^{\infty} u_{k}(x) t^{k / q}\right) .
\end{aligned}
$$

Assuming we can interchange the summation and the fractional derivative operator and using property 2 above, we obtain

$$
\begin{aligned}
& \sum_{k=1}^{\infty} a_{k}(x) s_{k} t^{(k-p) / q} \\
& \quad=\sum_{k=0}^{\infty} a_{k}^{\prime \prime}(x) t^{k / q}+h\left(x, t, \sum_{k=0}^{\infty} a_{k}(x) t^{k / q}\right),
\end{aligned}
$$

where $s_{k}=\Gamma(k / q+1) / \Gamma(k / q-\alpha+1)$. Note that if $p>1$, we will have negative powers of $t$ (for $k=1,2, \ldots, p-1$ ) in the sum on the left hand side of (9). To avoid this, we multiply (9) by $t^{(p-1) / q}$ to get

$$
\begin{aligned}
\sum_{k=0}^{\infty} a_{k+1}(x) s_{k+1} t^{k / q}= & \sum_{k=0}^{\infty} a_{k}^{\prime \prime}(x) t^{(k+p-1) / q}+t^{(p-1) / q} h \\
& \times\left(x, t, \sum_{k=0}^{\infty} a_{k}(x) t^{k / q}\right) .
\end{aligned}
$$

One way of finding the coefficients $a_{k}(x)$, in line with finding the coefficients of a Taylor series, is to recursively apply the operator $D_{t}^{1 / q}$ to (10) and substitute $t=0$. However, this is not convenient for implementation. To avoid the use of the fractional differentiation, we introduce the change of variable $w=t^{1 / q}$ which transforms (10) into

$$
\begin{aligned}
\sum_{k=0}^{\infty} a_{k+1}(x) s_{k+1} w^{k}= & \sum_{k=0}^{\infty} a_{k}^{\prime \prime}(x) w^{k+p-1}+w^{p-1} h \\
& \times\left(x, w^{q}, \sum_{k=0}^{\infty} a_{k}(x) w^{k}\right) .
\end{aligned}
$$


Now, differentiating ordinarily $k$ times with respect to $w$ and substituting $w=0$, we find the following recursion relation for $k \geq 0$ :

$$
\begin{aligned}
a_{k+1}(x)= & \frac{1}{s_{k+1}} a_{k-p+1}^{\prime \prime}(x)+\frac{1}{s_{k+1} k !} \\
& \times\left[\frac{\partial^{k}}{\partial w^{k}}\left[w^{p-1} h\left(x, w^{q}, \sum_{m=0}^{\infty} a_{m}(x) w^{m}\right)\right]\right]_{w=0},
\end{aligned}
$$

where we assume that $a_{l}(x) \equiv 0$ for $l<0$.

We note that if problem (1) and (2) is an ordinary differential equation of fractional order, that is, $u \equiv u(t)$ and $h \equiv h(t, u)$, the coefficients $a_{k}$ are real numbers and the recursion relation (12) reduces to

$$
a_{k+1}=\frac{1}{s_{k+1} k !}\left[\frac{\partial^{k}}{\partial w^{k}}\left[w^{p-1} h\left(w^{q}, \sum_{m=0}^{\infty} a_{m} w^{m}\right)\right]\right]_{w=0} .
$$

Remark 1. We remark that the present method is different in many ways from the ADM. A main difference between the two methods is that the ADM, in its generation of successive terms, uses fractional integration while the present method uses ordinary differentiation. However, when $\alpha=1(p=q=$ 1), formula (13) will reduce to

$$
a_{k+1}=\frac{1}{(k+1) !}\left[\frac{\partial^{k}}{\partial w^{k}}\left[h\left(w, \sum_{m=0}^{\infty} a_{m} w^{m}\right)\right]\right]_{w=0},
$$

which is the well-known Adomian polynomial formula [32].

In Section 3, we present several examples to show the practicality of this approach and make a comparison with other techniques such as the Adomian decomposition method and homotopy perturbation method.

\section{Numerical Results}

Example 1. Consider the fractional initial value problem

$$
D_{t}^{1 / 2} y=\Gamma\left(\frac{3}{2}\right)\left(y^{2}-t+1\right), \quad y(0)=0,
$$

with $y(t)=t^{1 / 2}$ being the exact solution.

Applying the proposed algorithm, the solution takes the form $y=\sum_{k=0}^{\infty} a_{k} t^{k / 2}$. The zeroth coefficient is readily given by $a_{0}=y(0)=0$. For $k \geq 0$, we have from (13)

$$
\begin{aligned}
a_{k+1} & =\frac{\Gamma(3 / 2)}{s_{k+1} k !}\left[\frac{\partial^{k}}{\partial w^{k}}\left[\left(\sum_{m=0}^{\infty} a_{m} w^{m}\right)^{2}-w^{2}+1\right]\right]_{w=0} \\
& =\frac{\Gamma(3 / 2)}{s_{k+1} k !}\left[k ! \sum_{m=0}^{k} a_{m} a_{k-m}-2 \delta_{k-2}+\delta_{k}\right]
\end{aligned}
$$

where $\delta_{j}=1$ if $j=0$ and 0 otherwise. With $k=0$, (17) gives $a_{1}=\left(\Gamma(3 / 2) / s_{1}\right)\left(a_{0}^{2}+1\right)=1$, since $s_{1}=\Gamma(3 / 2)$. It can be easily verified that (17) gives $a_{k}=0$ for $k \geq 2$. Hence the solution is

$$
y(t)=\sum_{k=0}^{\infty} a_{k} t^{k / 2}=a_{1} t^{1 / 2}=t^{1 / 2}
$$

which is the exact solution.

First, we compare our results with the Adomian decomposition method (ADM). To apply the ADM, assume that the solution $y(t)$ of (15) and the nonlinear function $f(y)=$ $y^{2}$ can be written in the series form as

$$
y(t)=\sum_{n=0}^{\infty} y_{n}(t), \quad f(y)=\sum_{n=0}^{\infty} A_{n},
$$

where $A_{n}, n=0,1,2, \ldots$, are called the Adomian polynomials. These polynomials can be derived by expanding the function $f(y)$ about $y_{0}$ as follows:

$$
f(y)=f\left(y_{0}\right)+f^{\prime}\left(y_{0}\right) \frac{y-y_{0}}{1 !}+f^{\prime \prime}\left(y_{0}\right) \frac{\left(y-y_{0}\right)^{2}}{2 !}+\cdots
$$

or

$$
f(y)=f\left(y_{0}\right)+f^{\prime}\left(y_{0}\right) \frac{\sum_{n=1}^{\infty} y_{n}}{1 !}+f^{\prime \prime}\left(y_{0}\right) \frac{\left(\sum_{n=1}^{\infty} y_{n}\right)^{2}}{2 !}+\cdots .
$$

Thus, $A_{k}$ can be derived as

$$
A_{k}=\frac{1}{k !} \frac{d^{k}}{d \beta^{k}}\left[f\left(\sum_{j=0}^{\infty} \beta^{j} y_{j}\right)\right]_{\beta=0}, \quad j \geq 0 .
$$

Next, define the fractional differential operator $L$ as $L=$ $D_{t}^{1 / 2}$; then (15) can be written in the form

$$
L(y)=\Gamma\left(\frac{3}{2}\right)(f(y)-t+1) .
$$

And defining the inverse operator as $L^{-1}(\cdot)=I_{t}^{1 / 2}$, then the solution $y(t)$ of (15) can be written in the form

$$
\begin{aligned}
y(t) & =\Gamma\left(\frac{3}{2}\right) I_{t}^{1 / 2}(f(y))+\Gamma\left(\frac{3}{2}\right) I_{t}^{1 / 2}((-t+1)) \\
& =\Gamma\left(\frac{3}{2}\right) I_{t}^{1 / 2}\left(\sum_{n=0}^{\infty} A_{n}\right)+\Gamma\left(\frac{3}{2}\right) I_{t}^{1 / 2}((-t+1)) \\
& =\sum_{n=0}^{\infty} y_{n}(t) .
\end{aligned}
$$

Now balancing the last equality in (24) yields

$$
\begin{gathered}
y_{0}(t)=\Gamma\left(\frac{3}{2}\right) I_{t}^{1 / 2}(-t+1), \\
y_{k+1}(t)=\Gamma\left(\frac{3}{2}\right) I_{t}^{1 / 2}\left(A_{k}\right), \quad k=0,1, \ldots
\end{gathered}
$$


The first few terms generated by ADM are given below:

$$
\begin{gathered}
y_{0}(t)=\frac{1}{3}(3-2 t) t^{1 / 2} \\
y_{1}(t)=\frac{2}{3} t^{3 / 2}-\frac{32}{45} t^{5 / 2}+\frac{64}{315} t^{7 / 2} \\
y_{2}(t)=\frac{32}{45} t^{5 / 2}-\frac{1664}{1575} t^{7 / 2}+\frac{32768}{59535} t^{9 / 2}-\frac{65536}{654885} t^{11 / 2}
\end{gathered}
$$

Next, we will compare our results with the homotopy perturbation method (HPM). To apply the HPM, define the homotopy $H:[0, \infty] \times[0,1] \rightarrow \mathbb{R}$ which satisfies

$$
\begin{aligned}
H(y, p)= & (1-p) D^{1 / 2} y \\
& +p\left(D^{1 / 2} y-\Gamma\left(\frac{3}{2}\right) y^{2}-\Gamma\left(\frac{3}{2}\right)(1-t)\right)=0 .
\end{aligned}
$$

The basic assumption is that the solution of problem (15) can be expressed as a power series in $p$ :

$$
y=y_{0}+p y_{1}+p^{2} y_{2}+\cdots,
$$

where $y_{i}(0)=0$ for $i \geq 0$. The approximate solution of problem (15) can be obtained as

$$
y(t)=\lim _{p \rightarrow 1}\left(y_{0}+p y_{1}+p^{2} y_{2}+\cdots\right) .
$$

The convergence of the last series has been proved in [33].

Substituting (28) into (27) and equating the coefficients of the terms with like powers of $p$, we have

$$
\begin{gathered}
p^{0}: D^{1 / 2} y_{0}=0, \quad y_{0}(0)=0, \\
p^{1}: D^{1 / 2} y_{1}-\Gamma\left(\frac{3}{2}\right) y_{0}^{2}-\Gamma\left(\frac{3}{2}\right)(1-t)=0, \quad y_{1}(0)=0, \\
p^{2}: D^{1 / 2} y_{2}-2 \Gamma\left(\frac{3}{2}\right) y_{0} y_{1}=0, \quad y_{2}(0)=0, \\
p^{3}: D^{1 / 2} y_{3}-\Gamma\left(\frac{3}{2}\right)\left(y_{1}^{2}+2 y_{0} y_{2}\right)=0, \quad y_{3}(0)=0,
\end{gathered}
$$

which implies that

$$
\begin{gathered}
y_{0}(t)=0, \\
y_{1}(t)=\sqrt{t}-\frac{2}{3} t^{3 / 2}, \\
y_{2}(t)=0,
\end{gathered}
$$

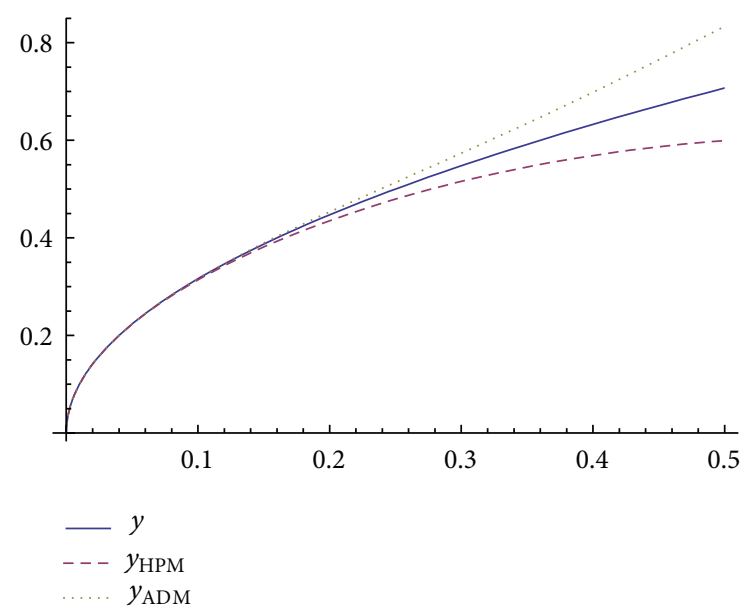

FIGURE 1: The exact and approximate solutions of Example 1 for $0 \leq$ $t \leq 0.5$.

$$
\begin{gathered}
y_{3}(t)=\frac{2}{3} t^{3 / 2}-\frac{32}{45} t^{5 / 2}+\frac{64}{315} t^{7 / 2}, \\
y_{4}(t)=0 \\
y_{5}(t)=\frac{32}{45} t^{5 / 2}-\frac{1664}{1575} t^{7 / 2}+\frac{32768}{59535} t^{9 / 2}-\frac{65536}{654885} t^{11 / 2} .
\end{gathered}
$$

The approximate solution is

$$
y_{\mathrm{HPM}}(t)=\sqrt{t}-\frac{64}{75} t^{7 / 2}+\frac{32768}{59535} t^{9 / 2}-\frac{65536}{654885} t^{11 / 2}+\cdots
$$

Figure 1 depicts the exact solution and the approximate solutions $y_{\mathrm{ADM}}(t)=\sum_{k=0}^{4} y_{k}(t)$ and $y_{\mathrm{HPM}}(t)=\sum_{k=0}^{4} y_{k}(t)$ obtained by the Adomian decomposition method and the homotopy perturbation method, respectively.

Example 2. Consider the fractional initial value problem

$$
D_{t}^{2 / 3} y=\frac{1}{2 \Gamma(4 / 3)} t^{1 / 3}(-3 \sqrt{y}-1), \quad y(0)=1,
$$

with $y(t)=(t-1)^{2}$ being the exact solution.

Here $\alpha=2 / 3$; hence $p=2$ and $q=3$. The solution assumes the form $y=\sum_{n=0}^{\infty} a_{n} t^{n / 3}$ with $a_{0}=y(0)=1$. Then, according to the previous section, we have for $k \geq 0$

$$
\begin{aligned}
a_{k+1}= & \frac{1}{2 \Gamma(4 / 3) s_{k+1} k !} \\
& \times\left[\frac{\partial^{k}}{\partial w^{k}} w^{2}\left(-3\left[\sum_{m=0}^{\infty} a_{m} w^{m}\right]^{1 / 2}-1\right)\right]_{w=0} .
\end{aligned}
$$

Numerical computation of (34) gives $a_{3}=-2, a_{6}=1$, and all other coefficients are zero. Thus, our procedure produces the solution

$$
y(t)=a_{0}+a_{3} t+a_{6} t^{2}=1-2 t+t^{2}=(t-1)^{2},
$$

which is the exact solution. 
TABLE 1: Error for various $t$ values and different values of $n$ for Example 3 .

\begin{tabular}{lccccc}
\hline$n$ & $t=0.2$ & $t=0.4$ & $t=0.6$ & $t=0.7$ & $t=1.0$ \\
\hline 5 & 0.001798 & 0.0136945 & 0.0443229 & 0.101588 & 0.193623 \\
10 & $3.2139 \times 10^{-6}$ & 0.0000989578 & 0.000725096 & 0.00295596 & 0.00874915 \\
15 & $2.70532 \times 10^{-10}$ & $6.70364 \times 10^{-8}$ & $1.66634 \times 10^{-6}$ & 0.000016172 & 0.0000938036 \\
20 & $1.30212 \times 10^{-13}$ & $1.29792 \times 10^{-10}$ & $7.29593 \times 10^{-9}$ & $1.26453 \times 10^{-7}$ & $1.15074 \times 10^{-6}$ \\
\hline
\end{tabular}
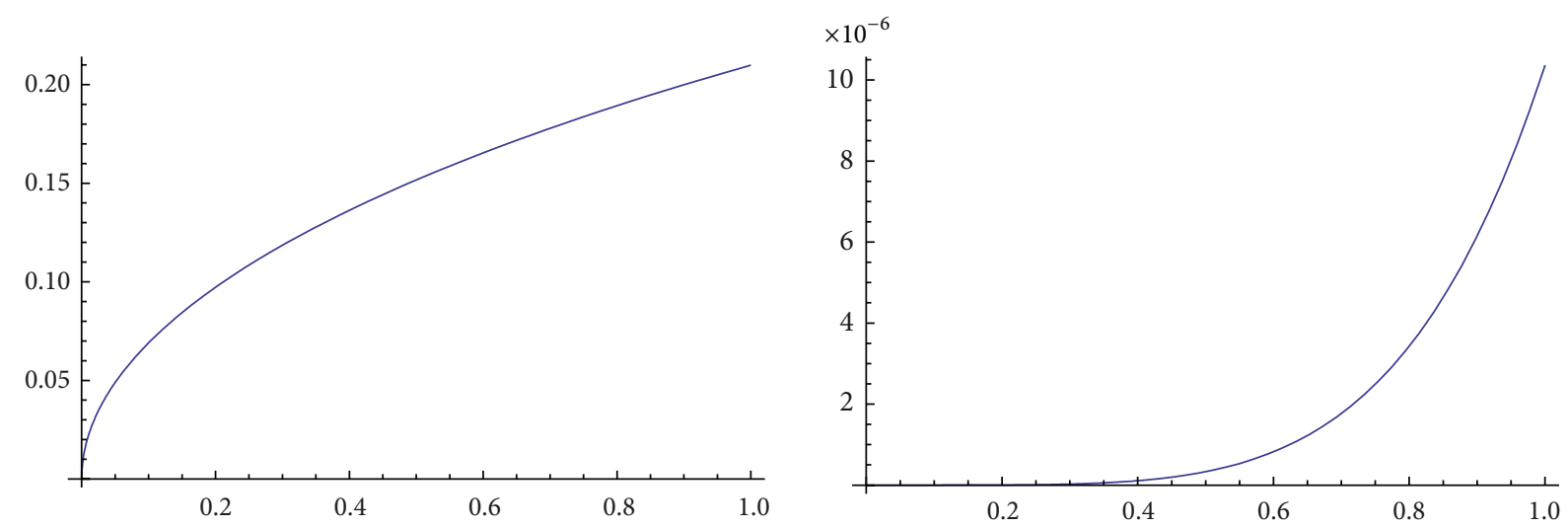

Figure 2: A plot of $y_{10}$ and $E_{10}$, for Example 3, where $0 \leq t \leq 1$.

Example 3. Consider the fractional initial value problem

$$
D_{t}^{\alpha} y=1-y^{2}, \quad y(0)=0 .
$$

For $\alpha=p / q$, the solution assumes the form $y=$ $\sum_{k=0}^{\infty} a_{k} t^{k / q}$ with $a_{0}=y(0)=0$. Then according to the previous section, we have for $k \geq 0$

$$
\begin{aligned}
a_{k+1} & =\frac{1}{s_{k+1} k !}\left[\frac{\partial^{k}}{\partial w^{k}} w^{p-1}\left(1-\left[\sum_{m=0}^{\infty} a_{m} w^{m}\right]^{2}\right)\right]_{w=0} \\
& =\frac{1}{s_{k+1} k !}\left[\frac{\partial^{k}}{\partial w^{k}} w^{p-1}\left(1-\sum_{m=0}^{\infty} C_{m} w^{m}\right)\right]_{w=0},
\end{aligned}
$$

where

$$
C_{m}=\sum_{j=0}^{m} a_{j} a_{m-j} .
$$

Simplification of (38) reveals the following recursion, where $k \geq 0$ :

$$
a_{k+1}= \begin{cases}0, & \text { if } k<p-1 \\ \frac{\delta_{k-p+1}}{s_{k+1}}-\frac{1}{s_{k+1}} C_{k-p+1}, & \text { if } k \geq p-1 .\end{cases}
$$

Numerical computation of (38) gives $a_{2 j}=0, j \geq 0$. We consider $\alpha=1 / 2$. Since the exact solution, in closed form, is not available, we define the error

$$
E_{n}(t)=\left|D_{t}^{1 / 2} y_{n}-\left(1-y_{n}^{2}\right)\right|
$$

where $y_{n}=\sum_{k=0}^{n} a_{k} t^{k / q}$. Figure 2 on the left presents the approximate solution $y_{10}=\sum_{k=0}^{10} a_{k} t^{k / 2}$ and on the right the error $E_{10}=\left|D_{t}^{1 / 2} y_{10}-\left(1-y_{10}^{2}\right)\right|$. Table 1 depicts the absolute error $E_{n}$ for different values of $n$ at various values of $t$. From the results presented, it is clear that the series converges and sufficient accuracy is achieved with few terms. However, more terms would be needed for larger values of $t$ which is expected for any initial value problem. We note that, for $\alpha=1$, we get $s_{k}=k$ and $a_{1}=1, a_{3}=-1 / 3, a_{5}=2 / 15, a_{7}=-17 / 315, a_{9}=$ $62 / 2835, \ldots$, and the obtained series solution is

$$
y(t)=t-\frac{1}{3} t-\frac{1}{3} t^{3}+\frac{2}{15} t^{5}-\frac{17}{315} t^{7}+\frac{62}{2835} t^{9}+\cdots
$$

which coincides with the Taylor series expansion of the exact solution $y(t)=\left(e^{2 t}-1\right) /\left(e^{2 t}+1\right)$.

Example 4. Consider the fractional initial value problem

$$
D_{t}^{\alpha} u=u_{x x}+\frac{1}{10} u(1-u), \quad u(x, 0)=x
$$

For this example we take $\alpha=1 / 2$; hence $p=1$ and $q=2$. The solution assumes the form $y=\sum_{k=0}^{\infty} a_{k}(x) t^{k / 2}$ with $a_{0}(x)=u(x, 0)=x$. Then from (12), we have, for $k \geq 0$,

$$
\begin{aligned}
a_{k+1}(x)= & \frac{1}{s_{k+1}} a_{k-p+1}^{\prime \prime}(x)+\frac{1}{s_{k+1} k !} \\
& \times\left[\frac{\partial^{k}}{\partial w^{k}}\left[w^{p-1} h\left(w^{q}, x, \sum_{m=0}^{\infty} a_{m}(x) w^{m}\right)\right]\right]_{w=0},
\end{aligned}
$$


TABLE 2: Error for various $x$ and $t$ values and $n=5$.

\begin{tabular}{lcccc}
\hline$x$ & $t=0.2$ & $t=0.4$ & $t=0.6$ & $t=0.8$ \\
\hline 0 & $8.3726110 \times 10^{-6}$ & 0.0000474301 & 0.000130878 & 0.000269023 \\
0.5 & $2.38184 \times 10^{-9}$ & $3.53911 \times 10^{-8}$ & $1.69571 \times 10^{-7}$ & $5.09316 \times 10^{-7}$ \\
1 & $8.2742 \times 10^{-6}$ & 0.000046751 & 0.000128695 & 0.000263925 \\
\hline
\end{tabular}

TABLE 3: Error for various $x$ and $t$ values and $n=15$.

\begin{tabular}{lcccc}
\hline$x$ & $t=0.2$ & $t=0.4$ & $t=0.6$ & $t=0.8$ \\
\hline 0 & $3.59581 \times 10^{-11}$ & $6.5209 \times 10^{-9}$ & $1.36682 \times 10^{-7}$ & $1.18428 \times 10^{-6}$ \\
0.5 & $1.3542 \times 10^{-12}$ & $2.39819 \times 10^{-10}$ & $4.93086 \times 10^{-9}$ & $4.19941 \times 10^{-8}$ \\
1 & $3.53848 \times 10^{-11}$ & $6.40568 \times 10^{-9}$ & $1.34033 \times 10^{-7}$ & $1.15927 \times 10^{-6}$ \\
\hline
\end{tabular}

$$
\begin{aligned}
= & \frac{1}{s_{k+1}} a_{k}^{\prime \prime}(x)+\frac{1}{10 s_{k+1} k !} \\
& \times\left[\frac{\partial^{k}}{\partial w^{k}}\left[\sum_{m=0}^{\infty} a_{m}(x) w^{m}-\sum_{m=0}^{\infty} C_{m}(x) w^{m}\right]\right]_{w=0}, \\
= & \frac{1}{s_{k+1}} a_{k}^{\prime \prime}(x)+\frac{1}{10 s_{k+1}}\left(a_{k}(x)-C_{k}(x)\right),
\end{aligned}
$$

where

$$
C_{k}(x)=\sum_{j=0}^{k} a_{j}(x) a_{k-j}(x) .
$$

The first few terms of the series solution are

$$
\begin{gathered}
u(x, t)=x+\frac{\sqrt{t}}{\sqrt{\pi}}\left(\frac{262144}{4849845} x-\frac{262144}{4849845} x^{2}\right) \\
+\frac{t}{\pi}\left(-\frac{274877906944}{4704199304805}+\frac{68719476736}{23520996524025} x\right. \\
-\frac{68719476736}{7840332174675} x^{2} \\
\left.+\frac{137438953472}{23520996524025} x^{3}\right) \\
+\left(\frac{t}{\pi}\right)^{3 / 2}\left(-\frac{288230376151711744}{22814637477412005225}\right. \\
+\frac{414331165718085632}{16296169626722860875} x \\
\quad-\frac{36028797018963968}{38024395795686675375} x^{2} \\
+\frac{36028797018963968}{22814637477412005225} x^{3} \\
\left.-\frac{18014398509481984}{22814637477412005225} x^{4}\right)+\cdots
\end{gathered}
$$

Tables 2 and 3 present the error

$$
E_{n}(x, t)=\left|D_{t}^{1 / 2} u_{n}-u_{n x x}-\frac{1}{10} u_{n}\left(1-u_{n}\right)\right|
$$

for various values of $t$ and $x$ and $n=5,15$, where $u_{n}(x, t)=$ $\sum_{k=0}^{n} a_{k}(x) t^{k / 2}$. The presented data indicate the accuracy of the series solutions obtained.

\section{Concluding Remarks}

We have presented a new algorithm for obtaining a series solution for a class of fractional differential equations. The algorithm is developed for a class of fractional partial differential equations of the Caputo type. We have applied the new algorithm to different examples. Accurate numerical solutions have been obtained as well as exact solutions for certain problems. The new algorithm is compared with the two well-known methods, the Adomian decomposition method (ADM) and the homotopy perturbation method (HPM), for one example. The exact solution is obtained after one step in the current method and after getting a telescoping sum by the HAM, where an approximate solution is obtained by the ADM. The idea of the new algorithm can be generalized to deal with various types of fractional functional equations.

\section{Conflict of Interests}

The authors declare that there is no conflict of interests regarding the publication of this paper.

\section{Acknowledgments}

This research is supported by the Individual Research Grant 21S074. The authors would like to express their sincere appreciation to the Research Affairs at the United Arab Emirates University.

\section{References}

[1] J. H. He, "Approximate analytical solution for seepage flow with fractional derivatives in porous media," Computer Methods in Applied Mechanics and Engineering, vol. 167, no. 1-2, pp. 57-68, 1998.

[2] J. H. He, "Some applications of nonlinear fractional differential equations and their approximations," Bulletin of Science, Technology \& Society, vol. 15, no. 2, pp. 86-90, 1999. 
[3] F. Mainardi, "Fractional calculus: some basic problems in continum and statistical mechanics," in Fractals and Fractional Calculus in Continuum Mechanics, pp. 291-348, Springer, New York, NY, USA, 1997.

[4] G. O. Young, "Definition of physical consistent damping laws with fractional derivatives," Zeitschrift für Angewandte Mathematik und Mechanik, vol. 75, no. 8, pp. 623-635, 1995.

[5] D. Baleanu, J. A. Machado, C. Cattani, M. C. Baleanu, and X. J. Yang, "Local fractional variational iteration and decomposition methods for wave equation on cantor sets within local fractional operators," Abstract and Applied Analysis, vol. 2014, Article ID 535048, 6 pages, 2014.

[6] H. Jafari and C. M. Khalique, "Homotopy perturbation and variational iteration methods for solving fuzzy differential equations," Communications in Fractional Calculus, vol. 3, no. $1,2012$.

[7] G.-C. Wu and D. Baleanu, "Variational iteration method for the Burgers flow with fractional derivatives: new Lagrange multipliers," Applied Mathematical Modeling, vol. 37, no. 9, pp. 6183-6190, 2013.

[8] X. J. Yang and D. Baleanu, "Fractal heat conduction problem solved by local fractional variation iteration method," Thermal Science, vol. 17, no. 2, pp. 625-628, 2013.

[9] S. Das and P. K. Gupta, "Approximate analytical solutions of time-space fractional diffusion equation by Adomian decomposition method and homotopy perturbation method," Communications in Fractional Calculus, vol. 2, no. 1, 2011.

[10] A. Kadem and D. Baleanu, "Homotopy perturbation method for the coupled fractional lotka-volterra equations," Romanian Journal in Physics, vol. 56, no. 3-4, pp. 332-338, 2011.

[11] Y.-M. Qin and D.-Q. Zeng, "Homotopy perturbation method for the q-diffusion equation with a source term," Communications in Fractional Calculus, vol. 3, no. 1, 2012.

[12] L. Song and H. Zhang, "Application of homotopy analysis method to fractional KdV-Burgers-Kuramoto equation," Physics Letters A, vol. 367, no. 1-2, pp. 88-94, 2007.

[13] N. H. Sweilam, M. M. Khader, and R. F. Al-Bar, "Numerical studies for a multi-order fractional differential equation," Physics Letters A, vol. 371, no. 1-2, pp. 26-33, 2007.

[14] H. Xu, "Analytical approximations for a population growth model with fractional order," Communications in Nonlinear Science and Numerical Simulation, vol. 14, no. 5, pp. 1978-1983, 2009.

[15] V. Daftardar-Gejji and S. Bhalekar, "Solving multi-term linear and non-linear diffusion-wave equations of fractional order by Adomian decomposition method," Applied Mathematics and Computation, vol. 202, no. 1, pp. 113-120, 2008.

[16] J.-S. Duan, R. Rach, D. Baleanu, and A.-M. Wazwaz, "A review of the adomian decomposition method and its applications to fractional differential equations," Communications in Fractional Calculus, vol. 3, no. 2, pp. 73-99, 2012.

[17] H. Jafari and V. Daftardar-Gejji, "Solving linear and nonlinear fractional diffusion and wave equations by Adomian decomposition," Applied Mathematics and Computation, vol. 180, no. 2, pp. 488-497, 2006.

[18] C. Yang and J. Hou, "An approximate solution of nonlinear fractional differential equation by Laplace transform and Adomian polynomials," Journal of Information and Computational Science, vol. 10, no. 1, pp. 213-222, 2013.

[19] X. J. Yang, D. Baleabu, and W.-P. Zhong, "Approximate solutions for diffusion equations on cantor space-time," Proceedings of the Romanian Academy A, vol. 14, no. 2, pp. 127-133, 2013.
[20] D. Q. Zeng and Y.-M. Qin, "The Laplace-Adomian-Pade technique for the seepage flows with the Riemann-Liouville derivatives," Communications in Fractional Calculus, vol. 3, no. $1,2012$.

[21] D. Baleanu, G. Wu, and J. Duan, "Some analytical techniques in fractional calculus: realities and challenges," in Discontinuity and Complexity in Nonlinear Physical Systems, Nonlinear Systems and Complexity, vol. 6, pp. 35-62, 2014.

[22] M. Al-Refai and M. Ali Hajji, "Monotone iterative sequences for nonlinear boundary value problems of fractional order," Nonlinear Analysis, Theory, Methods and Applications, vol. 74, no. 11, pp. 3531-3539, 2011.

[23] J. T. Chern, Finite element modeling of viscoelastic materials on the theory of fractional calculus [Ph.D. thesis], Pennsylvania State University, State College, Pa, USA, 1993.

[24] K. Diethelm, "An algorithm for the numerical solution of differential equations of fractional order," Electronic Transactions on Numerical Analysis, vol. 5, pp. 1-6, 1997.

[25] K. Diethelm and Y. Luchko, "Numerical solution of linear multi-term initial value problems of fractional order," Journal of Computational Analysis and Applications, vol. 6, no. 3, pp. 243263, 2004.

[26] C. Lubich, "On the stability of linear multistep methods for volterra convolution equations," IMA Journal of Numerical Analysis, vol. 3, no. 4, pp. 439-465, 1983.

[27] C. Lubich, "Fractional linear multistep methods for AbelVolterra integral equations of the second kind," Mathematics of Computation, vol. 45, no. 172, pp. 463-469, 1985.

[28] E. Hairer, C. Lubich, and M. Schlichte, "Fast numerical solution of nonlinear Voltera convolution equations," SIAM Journal on Scientific and Statistical Computing, vol. 6, no. 3, pp. 532-541, 1985.

[29] M. Weilbeer, Efficient Numerical Methods for Fractional Differential Equations and Their Analytical Background, Eingereicht: 23.01.2005, Prufung: 09.06.2005, Papierflieger, 2005.

[30] I. Podlubny, "Matrix approach to discrete fractional calculus," Fractional Calculus and Applied Analysis, vol. 3, no. 4, pp. 359$386,2000$.

[31] I. Podlubny, A. Chechkin, T. Skovranek, Y. Chen, and B. M. Vinagre Jara, "Matrix approach to discrete fractional calculus II: partial fractional differential equations," Journal of Computational Physics, vol. 228, no. 8, pp. 3137-3153, 2009.

[32] G. Adomian, Solving Frontier Problems of Physics: The Decomposition Method, Kluwer Academic, Dordecht, The Netherlands, 1994.

[33] J. H. He, "Coupling method of a homotopy technique and a perturbation technique for non-linear problems," International Journal of Non-Linear Mechanics, vol. 35, no. 1, pp. 37-43, 2000. 


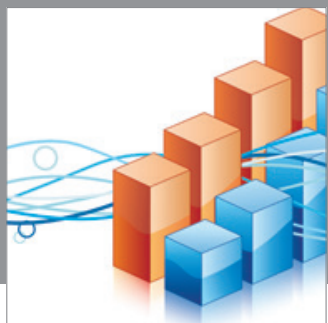

Advances in

Operations Research

mansans

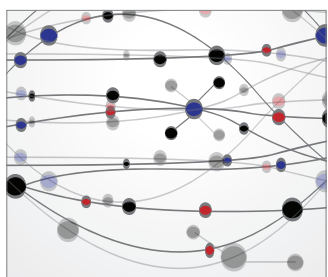

The Scientific World Journal
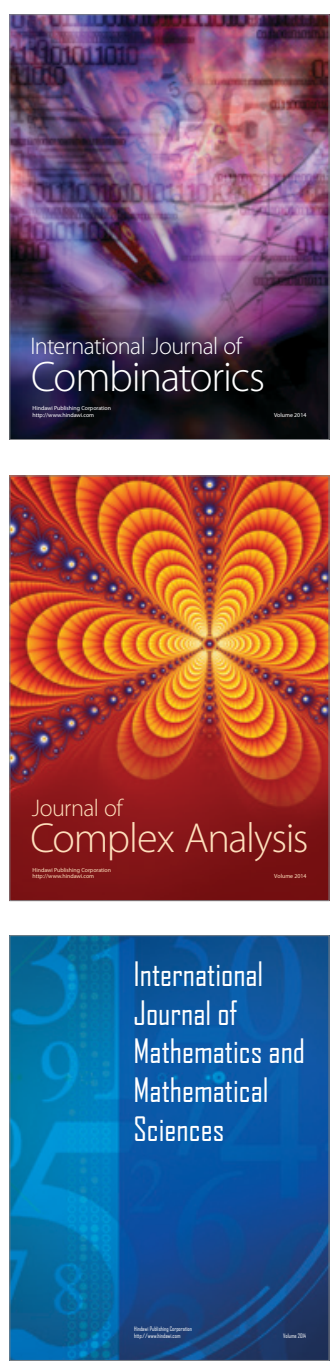
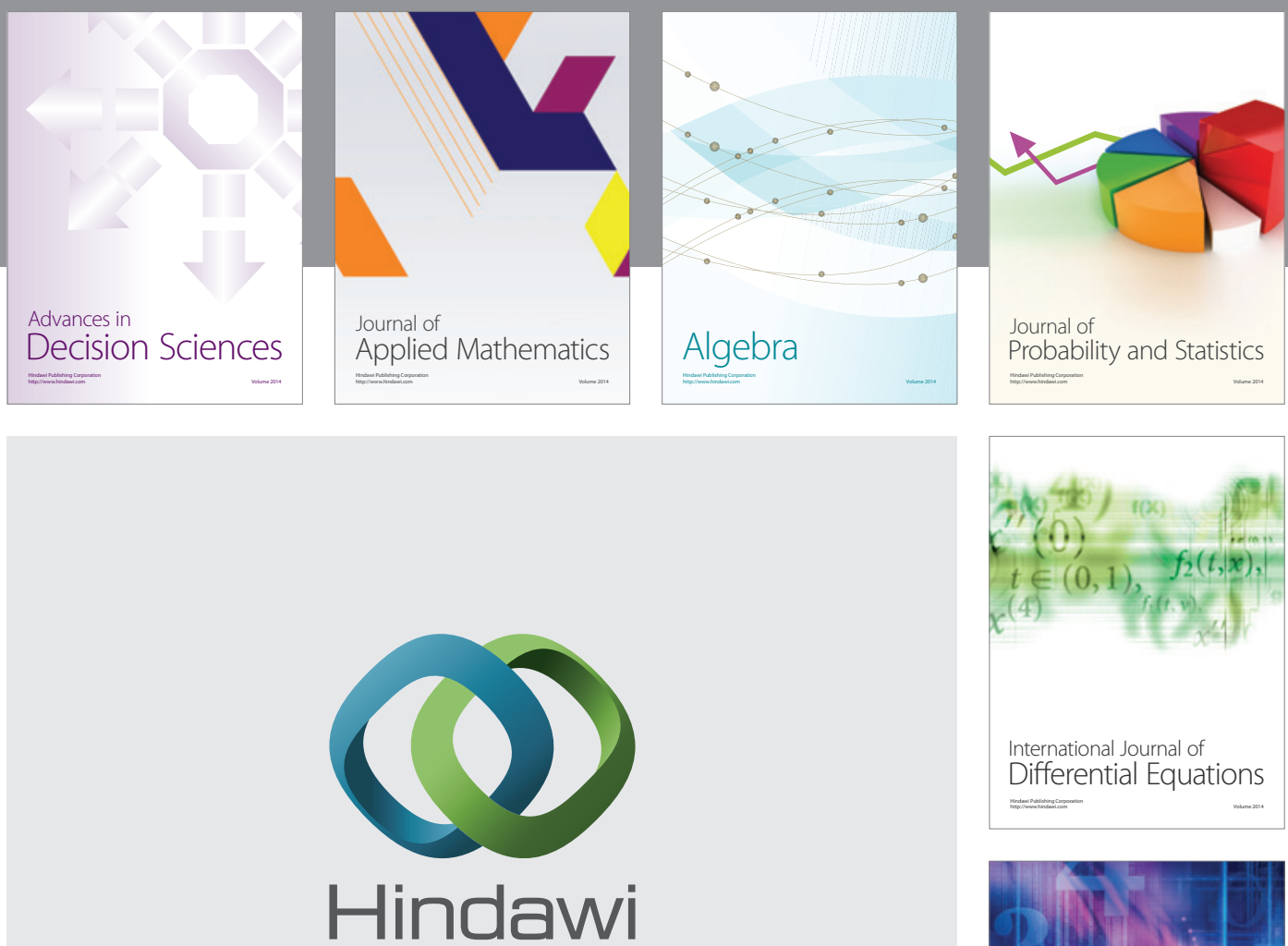

Submit your manuscripts at http://www.hindawi.com
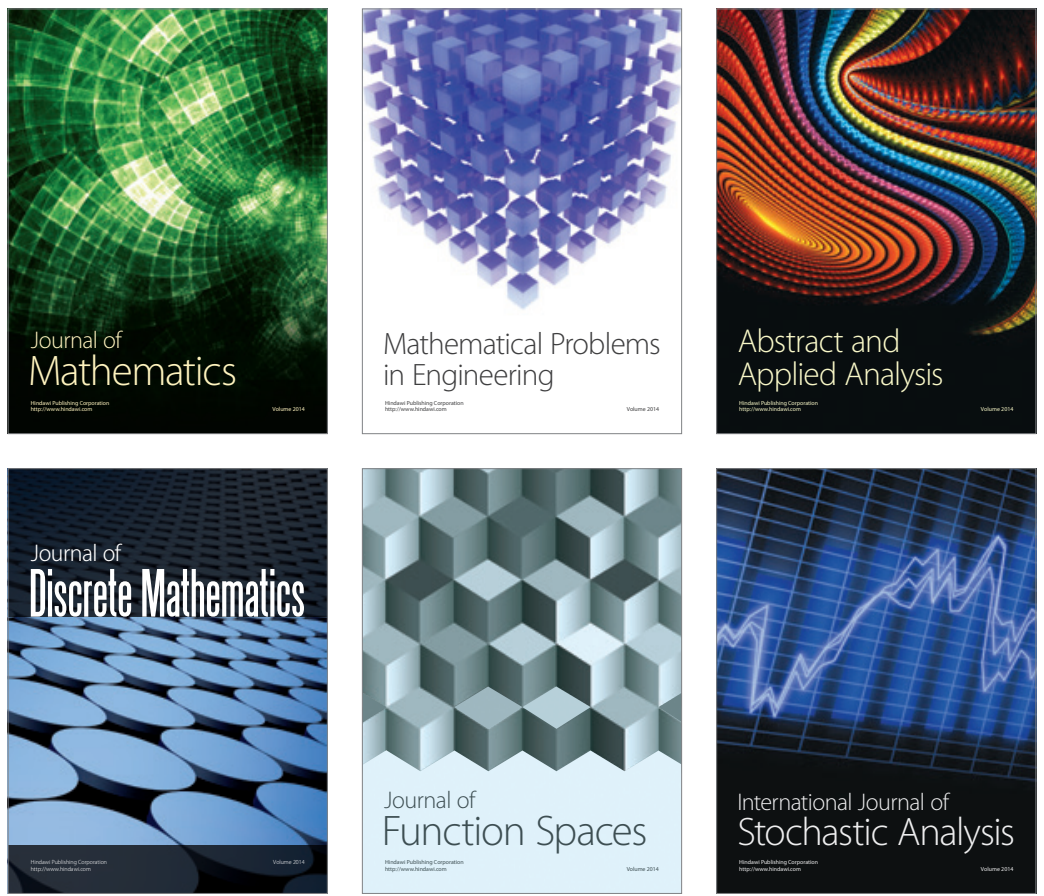

Journal of

Function Spaces

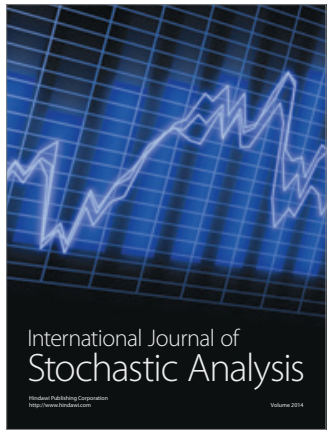

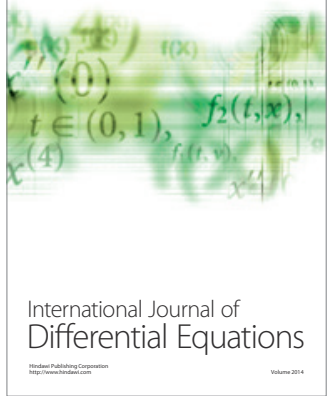
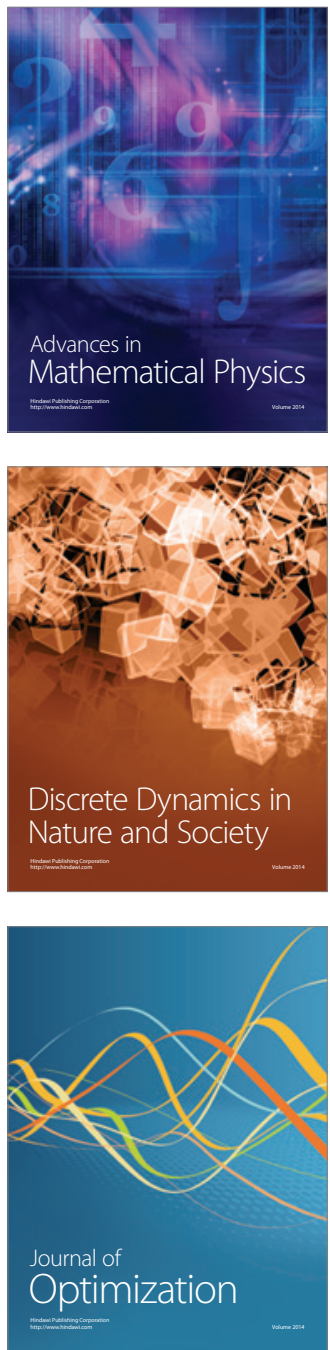